\title{
Biliary cystic tumors with bile duct communication: a cystic variant of intraductal papillary neoplasm of the bile duct
}

Yoh Zen ${ }^{1,2}$, Takahiko Fujii ${ }^{1}$, Keita Itatsu ${ }^{1}$, Koichi Nakamura ${ }^{1}$, Fumio Konishi ${ }^{3}$, Shinji Masuda ${ }^{4}$, Takeshi Mitsui ${ }^{5}$, Yasuyuki Asada ${ }^{5}$, Shouji Miura ${ }^{5}$, Shiro Miyayama ${ }^{6}$, Takeshi Uehara ${ }^{7}$, Tsutomu Katsuyama ${ }^{7}$, Tetsuo Ohta ${ }^{8}$, Hiroshi Minato ${ }^{2}$ and Yasuni Nakanuma ${ }^{1}$

${ }^{1}$ Department of Human Pathology, Kanazawa University Graduate School of Medicine, Kanazawa, Japan; ${ }^{2}$ Division of Pathology, Kanazawa University Hospital, Kanazawa, Japan; ${ }^{3}$ Department of Pathology, Fukui Red Cross Hospital, Fukui, Japan; ${ }^{4}$ Department of Pathology, Kouseiren Takaoka Hospital, Takaoka, Japan; ${ }^{5}$ Department of Surgery, Fukui Saiseikai Hospital, Fukui, Japan; ${ }^{6}$ Department of Radiology, Fukui Saiseikai Hospital, Fukui, Japan; ${ }^{7}$ Department of Laboratory Medicine, Shinshu University School of Medicine, Matsumoto, Japan and ${ }^{8}$ Department of Gastroenterologic Surgery, Kanazawa University Graduate School of Medicine, Kanazawa, Japan

Biliary cystic tumors, which are also called biliary cystadenoma and cystadenocarcinoma, are thought to be a heterogeneous disease entity, and some of them are known to show a luminal communication to the bile duct. In this study, we examined the clinicopathological features of nine cases of biliary cystic tumors with bile duct communication. They were composed of five males and four females with an average age of 67 years (52-84 years). They were multilocular (eight cases) or unilocular (one case), and all cases contained mucinous fluid. A direct luminal communication with the bile ducts was identified in five cases on preoperative or intraoperative cholangiographies. Biliary cystic tumors examined in this study were histologically adenoma (one case), adenocarcinoma in situ (six cases), and adenocarcinoma associated with microinvasive mucinous carcinoma (two cases). One case of adenocarcinoma in situ also had the adenoma component (adenocarcinoma in adenoma). Dysplastic mucinous epithelium proliferated in flat, micropapillary and papillary fashions within the intracystic spaces. Intraepithelial neoplasm was observed within non-dilated adjacent bile ducts, suggesting a direct luminal communication between the cystic tumors and the bile duct. Ovarian-like stroma was not observed in their walls in any cases. Immunohistochemically, seven cases expressed MUC1 or MUC2 in the neoplastic biliary epithelium. All cases except one were alive without any evidences of tumor recurrence after total excision (3-156 months after surgery). These clinicopathological features resembled those of intraductal papillary neoplasm of the bile duct, which had been reported as a biliary counterpart of pancreatic intraductal papillary mucinous neoplasm. In conclusion, biliary cystic tumors with bile duct communication could be regarded as intraductal papillary neoplasm with a prominent cystic dilatation of the bile duct and mucin retention, rather than true biliary cystic neoplasms.

Modern Pathology (2006) 19, 1243-1254. doi:10.1038/modpathol.3800643; published online 2 June 2006

Keywords: cholangiocarcinoma; intraductal papillary mucinous neoplasm; papillomatosis; dysplasia; MUC; mucin-producing bile duct tumor

Biliary cystic tumors are rare neoplasms occurring in the liver and less frequently in the extrahepatic biliary system. ${ }^{1,2}$ They account for less than $5 \%$ of the reported cystic lesions of the liver. ${ }^{3,4}$ According

Correspondence: Dr Y Nakanuma, MD, Department of Human Pathology, Kanazawa University Graduate School of Medicine, 13-1 Takaramachi, Kanazawa 920-8640, Japan.

E-mail: pbcpsc@kenroku.kanazawa-u.ac.jp

Received 15 March 2006; revised 5 May 2006; accepted 8 May 2006; published online 2 June 2006 to WHO classification of tumors, biliary cystic tumors are called biliary cystadenoma and cystadenocarcinoma, which are not defined histologically in detail but rather more clinically defined by cystic tumor lined by neoplastic biliary epithelium without any preceding conditions such as cystic congenital malformation. ${ }^{1}$ Therefore, biliary cystic tumors are histologically heterogeneous with regard to tumor cell types (mucinous or non-mucinous) and tumor stroma (presence or absence of ovarianlike stroma). ${ }^{1-4}$ The most common type is mucinous 
cystadenoma with ovarian-like stroma occurring only in females. ${ }^{4}$ Most of the reported cases had no luminal communication between cystic tumors and the bile duct, although recent advances in the imaging modalities revealed a direct luminal communication to the bile duct in some cases. ${ }^{5-7}$ However, it has not been clarified whether biliary cystic tumors with or without bile duct communication might reflect a distinct tumor or a different manifestation of the same tumor.

In the pancreas, two types of cystic neoplasms resembling biliary cystic tumors could occur. The first is mucinous cystic neoplasms, ${ }^{8}$ and the other is intraductal papillary mucinous neoplasm of the pancreas (IPMN-P). ${ }^{9}$ While there had been some confusion and controversy on clinical distinction between these two tumors in earlier times, ${ }^{10,11}$ the clinical and pathological differentiations of these two tumors are now accepted worldwide after reaching the definition of pancreatic mucinous cystic neoplasms. ${ }^{12,13}$ That is, mucinous cystic neoplasms of the pancreas were pathologically defined as having ovarian-like stroma, and those tumors usually occur in females and do not have a luminal communication with the pancreatic duct. ${ }^{8}$ In contrast, IPMN-P, which does not have ovarianlike stroma, occurs in both genders and has a communication to the pancreatic ductal system. ${ }^{9}$ That is, most cases previously reported as pancreatic mucinous cystic neoplasms with a luminal communication to the pancreatic duct or occurring in male patients are now thought to be IPMN-P. ${ }^{14,15}$

Recently, great attention is being drawn to intraductal biliary tumors. In 2001, we reported a characteristic intraductal papillary tumor of the bile duct associated with hepatolithiasis as a biliary counterpart of IPMN-P (intraductal papillary neoplasm of the bile duct: IPN-B). ${ }^{16}$ After that, it was reported that IPN-B could occur in the bile ducts without any preceding conditions. ${ }^{17,18}$ IPN-B was pathologically characterized by prominent intraductal papillary proliferation with delicate fibrovascular cores, the acquisition of gastro-intestinal phenotypes, frequent mucin hypersecretion, and common association with invasive mucinous carcinoma. ${ }^{19,20}$ Tumor-cell immunophenotypes and their alteration during tumor progression resembled those of IPMN-P, which supported a close relationship between IPN-B and IPMN-P. ${ }^{20}$ IPN-B was usually associated with local dilatation of the affected bile duct. Those experiences of IPN-B cases prompted us to test whether some cases of biliary cystic tumors, especially those having bile duct communication, might belong to IPN-B.

In this study, we examined the clinicopathological characteristics of biliary cystic tumors with bile duct communication to verify our hypothesis that those tumors with a ductal communication might be a cystic variant of IPN-B.

\section{Materials and methods}

\section{Case Selection}

A total of nine cases of biliary cystic tumor were obtained from the hepatobiliary disease files of the Department of Human Pathology, Kanazawa University Graduate School of Medicine and affiliated hospitals in Japan between 1994 and 2005. Biliary cystic tumor used in this study was found to show a luminal communication to the bile ducts on radiological images or pathological examinations (described in the Result Section). Ages and genders are shown in Table 1 . No cases had any preceding biliary diseases, such as primary sclerosing cholangitis, hepatolithiasis, choledochal cyst or Caroli's disease. All cases used in this study were surgically resected cases (five cases, left hepatectomy; two cases, segmentectomy; one case, right hepatectomy; and one case, left hepatectomy with upper choledochectomy).

\section{Pathological Examination}

Surgically resected material was macroscopically examined after immersion in formalin and parallel incision at $5-\mathrm{mm}$ intervals. We examined the

Table 1 Clinical features and survival status of 9 cases of biliary cystic tumor examined in this study

\begin{tabular}{llllll}
\hline Case & Age/gender & Clinical presentation & Tumor location & Treatment & Survival status $^{\mathrm{a}}$ \\
\hline 1 & $78 / \mathrm{M}$ & Epigastralgia & Left medial segment & Left lobectomy & Alive NED, 56 months \\
2 & $84 / \mathrm{M}$ & Epigastralgia & Left lateral segment & Segmentectomy & Alive, NED, 5 months \\
3 & $52 / \mathrm{F}$ & Deep in urinary color & Left medial segment & Left lobectomy & Alive, NED, 28 months \\
4 & $77 / \mathrm{M}$ & Anemia & Left lateral segment & Segmentectomy & Alive, NED, 3 months \\
5 & $83 / \mathrm{M}$ & Right hypochodrial pain & Left medial segment & Left lobectomy & Alive, NED, 15 months \\
6 & $72 / \mathrm{F}$ & Liver dysfunction & Right anterior segment & Right lobectomy & Alive, NED, 156 months \\
7 & $70 / \mathrm{F}$ & Epigastralgia & Left medial segment & Left lobectomy & Died, 1 month \\
8 & $66 / \mathrm{M}$ & Epigastralgia & Left medial segment & Left lobectomy & Alive, NED, 134 months \\
9 & $52 / \mathrm{F}$ & Epigastralgia & Left medial segment & $\begin{array}{l}\text { Left lobectomy with upper } \\
\text { choledochectomy }\end{array}$ & Alive, NED, 28 months \\
& & & and hepatic hilum & & \\
\hline
\end{tabular}

${ }^{\mathrm{a}}$ Duration after surgery.

NED, no evidence of disease. 
macroscopic characteristics of biliary cystic tumors with regard to cystic types (unilocular or multilocular), intracystic content, and an association with papillary or nodular lesions. A ductal communication was macroscopically examined using probes on surgically resected tissues. Next, formalin-fixed and paraffin-embedded specimens were prepared, and $4 \mu \mathrm{m}$ sections were cut for routine hematoxylin and eosin (H\&E) staining, mucin histochemical staining and immunohistochemical staining. The microscopic characteristics of biliary cystic tumors were examined with emphasis on tumor cell types, degrees of tumor cell atypia, papillary proliferation, mucin over-production and the possible association with invasive lesions. Biliary cystic tumors were considered to have a microscopic communication to the bile duct in that tumor cells were observed in the pre-existing bile ducts around the cystic tumors on histological specimens (intraductal spreading).

\section{Classification of Tumor-Cell Types}

We classified tumor cells into four types according to those of IPMN-P: ${ }^{21-24}$ (1) the pancreaticobiliary type, resembling biliary or pancreatic epithelium and composed of columnar cells with eosinophilic cytoplasm and round nuclei; (2) the intestinal type, resembling intestinal adenoma or adenocarcinoma characterized by stratified tall columnar cells with some goblet cells; (3) the gastric type, composed of columnar cells with abundant intracytoplasmic mucin; (4) the oncocytic type, characterized by abundant eosinophilic cytoplasm and round nuclei.

\section{Mucin Histochemistry}

Histochemical observations of mucin were carried out using periodic acid-Schiff staining after diastase digestion for neutral mucin and combined high-iron diamine and alcian blue ( $\mathrm{pH} 2.5)$ staining for acidic sulfomucin and sialomucin. Intracellular and extracellular mucin positive only for alcian blue was regarded as sialomucin, and that positive for highiron diamine with or without simultaneous alcian blue was regarded as sulfomucin. Mucin production on the periodic acid-Schiff, high-iron diamine and alcian blue staining was semiquantitatively classified into four categories on the basis of the percentage of positive cells: -(negative), $0 \% ; 1+$ (focal), 1 to $10 \% ; 2+$ (moderate), 11 to $50 \% ; 3+$ (extensive) more than $50 \%$.

\section{Immunohistochemistry}

Immunostainings of MUC1, MUC2, MUC5AC, carbohydrate antigen 19-9 (CA19-9), carcinoembryonic antigen (CEA), estrogen receptor (ER) and progesterone receptor (PgR) were performed using the
EnVision + system (Dako Cytomation, Glostrup, Denmark). Deparaffinized sections used for MUC2, MUC5AC, CEA, ER and PgR were microwaved in $10 \mathrm{mM}$ citrate buffer ( $\mathrm{pH}$ 6.0) for $20 \mathrm{~min}$. After the blocking of endogenous peroxidase and incubation in normal goat serum (1:10; Vector Laboratories, Burlingame, CA, USA) for $20 \mathrm{~min}$, the deparaffinized sections were incubated overnight at $4^{\circ} \mathrm{C}$ with primary monoclonal antibodies: MUC1 (clone DF3; 1:100; Toray Fuji Bionics, Tokyo, Japan), MUC2 (clone Ccp58; 1:100; Novocastra Laboratories, Newcastle, UK), MUC5AC (clone CLH2; 1:100; Novocastra Laboratories), CA19-9 (clone 1116NS19-9; 1:10 dilution; CIS bio international, France), CEA (clone II-7; 1:100; Dako Cytomation), ER (clone 1D5; 1:100 dilution; Dako Cytomation), and PgR (clone 16; 1:40 dilution; Novocastra Laboratories). The sections were then incubated at room temperature for $1 \mathrm{~h}$ with goat anti-mouse immunoglobulins conjugated to peroxidase labeled-dextran polymer (EnVision + ; Dako Cytomation). The reaction products were developed by immersing the section in a $3,3^{\prime}$-diaminobenzidine tetrahydrochloride (DAB) solution containing $0.03 \%$ hydrogen peroxide. Nuclei were lightly counterstained with hematoxylin.

The expression of MUC1, MUC2, MUC5AC, CA19-9 and CEA on tumor cells was evaluated semiquantitatively into four scores, according to the percentage of positive cells in the individual lesion: -(negative), $0 \% ; 1+$ (focal), 1 to $10 \% ; 2+$ (moderate), 11 to $50 \% ; 3+$ (extensive) more than $50 \%$. We examined nuclear expression of ER and PgR on subepithelial stromal cells.

\section{Double Immunostaining for CDX2 and MUC2}

Double immunohistochemical staining was performed to assess the simultaneous detection of CDX2 and MUC2 in neoplastic biliary epithelium. The deparaffinized sections were incubated with normal goat serum (1:10, Vector Laboratories) for $20 \mathrm{~min}$, immersed in $10 \mathrm{mM}$ citrate buffer and heated in a microwave oven at $95^{\circ} \mathrm{C}$ for $20 \mathrm{~min}$, and then incubated with mouse monoclonal antibody to CDX2 (clone CDX2-88; 1:100; BioGenex, San Ramon, CA, USA) and rabbit polyclonal antibody to MUC2 (clone H-300; 1:100; Santa Cruz Biotechnology, Santa Cruz, CA, USA) overnight at $4^{\circ} \mathrm{C}$. The reaction product was visualized with fluorescent goat anti-mouse or anti-rabbit IgG antibody (1:500, Molecular Probes Inc., Eugene, OR, USA), and observed under fluorescent microscopy.

\section{Statistical Analysis}

Statistical analysis was performed using the Mann-Whitney $U$-test. A probability of $P<0.05$ was considered statistically significant. 


\section{Results}

\section{Clinical and Radiological Characteristics}

Clinical features of nine cases of biliary cystic tumor are shown in Table 1. Biliary cystic tumors occurred almost equally in both genders (five males and four females). Epigastralgia was the commonest subjective symptom. Case 3 was jaundiced. Case 4 was found to have anemia during medical follow-up for cerebral hemorrhage. Case 6 was found to have serological liver dysfunction on a periodic medical examination. Serum levels of CA19-9 and CEA were within normal range in all cases.

All cases underwent radiological examinations, such as computed tomography (CT) and magnetic resonance imaging (MRI), and found to have multilocular (eight cases) or unilocular cystic tumors (one case) in the liver (Figure 1a and b). Mural nodules were also detected in every case on radiological images (Figure 1a). The left hepatic lobe was more commonly affected (Table 1). All cystic tumors were located within the liver, and a multilocular cystic tumor of case 3 protruded into the hepatic hilum (Figure 1b). Endoscopic retrograde cholangiography (ERC) revealed a luminal communication between cystic tumors and the bile ducts in four cases (cases 3, 6, 7 and 9) (Figure 1c). In addition, cases 7 and 8 underwent intraoperative cholangiography from cystic tumors (cystgraphy), which revealed a direct luminal communication between intracystic spaces and the bile duct in both cases. In case 3 , an intrahepatic cystic tumor with protrusion into the hepatic hilum was found to have a communication to the right hepatic duct on ERC. Mucinous discharge from Vater's ampulla was not observed on endoscopic examination during ERC in any cases. Surgical treatments were performed in all cases based on the radiological diagnosis of biliary cystadenoma or cystadenocarcinoma.

\section{Gross Findings}

Macroscopically, the average tumor size was $6.1 \mathrm{~cm}$ (range: $2.5-20.0 \mathrm{~cm}$ ). Eight cases were multilocular cystic tumors, and the remaining case (case 8) was a unilocular cystic tumor (Figure 2, Table 2). All cystic tumors contained mucinous fluid, which was associated with hemorrhage in two cases (cases 2 and 4). Except for mural nodules, the internal surfaces of the cystic tumors were generally smooth or finely granular. Papillary mural nodules, ranging from 0.5 to $3.2 \mathrm{~cm}$, were observed in all cases. Macroscopically, a communication between cystic tumors and the bile duct could not be confirmed in any of the cases, including cases 3, 6, 7, 8 and 9, which were found to have ductal communication on cholangiography, as mentioned above.

\section{Histological Findings}

Biliary cystic tumors were composed of the flat, micropapillary and papillary proliferation of dysplastic tumor cells (Figure 3). Prominent papillary proliferation with delicate fibrovascular cores was observed especially in mural nodules. Based on a tumor-cell classification of IPMN-P mentioned in the Materials and Methods, ${ }^{21-24}$ nine cases were classified into three types: cases 1 and 2 into the gastric type; cases $3,4,6,7,8$ and 9 into the pancreaticobiliary type; and case 5 into the oncocytic type (Figure 4 and Table 2). The intestinal type tumor was not observed in this study. The histological grade of each tumor is shown in Table 2. Two cases of the gastric type (cases 1 and 2) had adenoma components; and, in addition, case 2 was associated with non-invasive adenocarcinoma foci (adenocarcinoma in adenoma) (Figure 5). In contrast, seven cases of the pancreaticobiliary or oncocytic types were all histologically adenocarcinoma without
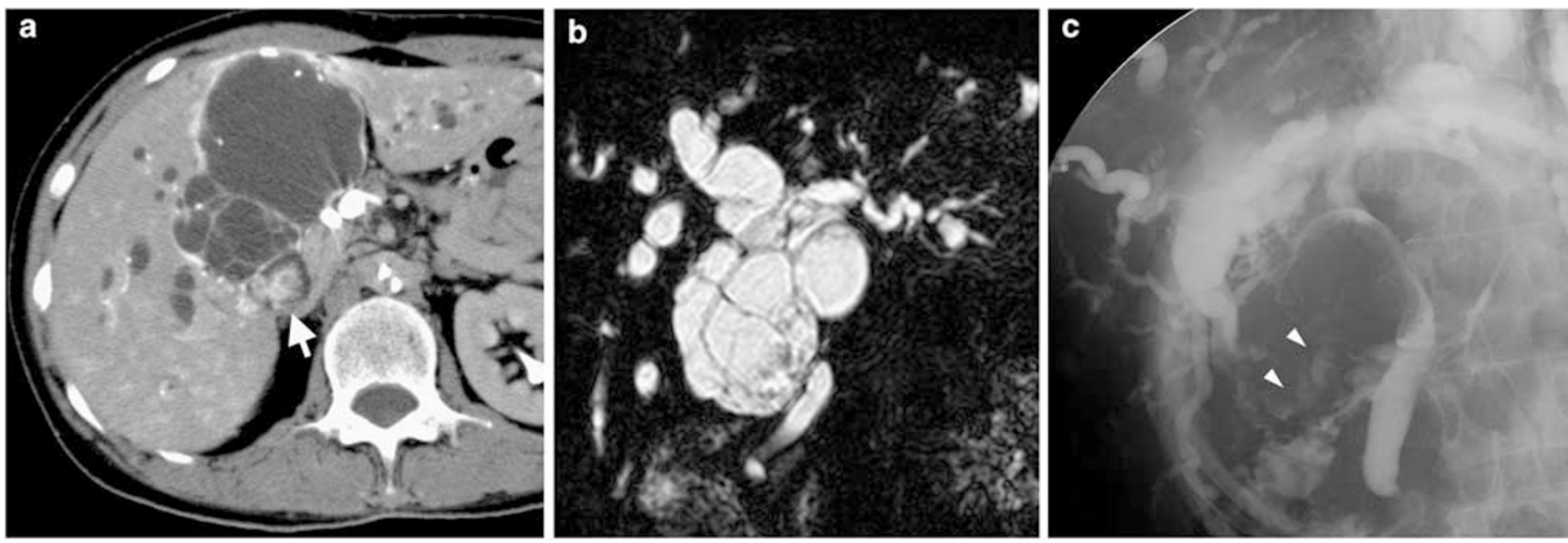

Figure 1 Radiological imagings of biliary cystic tumor. (a) Contrast-enhanced CT reveals multilocular cystic tumor with mural nodules (arrow). (b) Magnetic resonance cholangiography (MR cholangiography) reveals multilocular cystic tumor around the hepatic hilum. (c) Contrast medium is focally observed in the intracystic space (arrowheads) on endoscopic retrograde cholangiography, suggesting a communication between cystic tumor and biliary tract (case 3). 

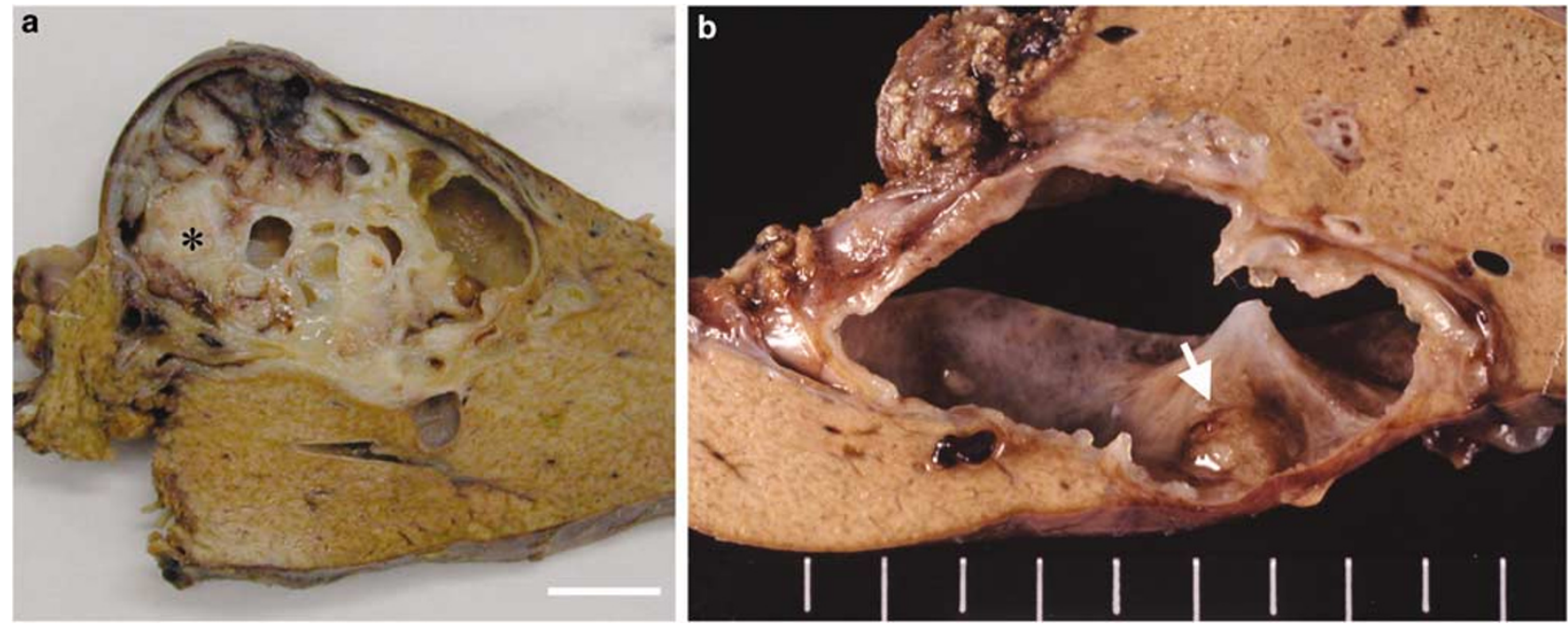

Figure 2 Macroscopic pictures of biliary cystic tumors. Multilocular (a) and unilocular cystic tumors (b) have intracystic papillary lesions (asterisk in a and arrow in b) (a: case 4, b: case 8, white bar: $1.0 \mathrm{~cm}$ ).

Table 2 Pathological and immunohistochemical characteristics of 9 cases of biliary cystic tumor

\begin{tabular}{|c|c|c|c|c|c|c|c|c|c|c|c|c|}
\hline Case & $\begin{array}{l}\text { Size } \\
(\mathrm{cm})\end{array}$ & $\begin{array}{l}\text { Type } \\
\text { of cyst }\end{array}$ & $\begin{array}{l}\text { Histological } \\
\text { diagnosis }\end{array}$ & Tumor-cell type & $P A S$ & $H I D$ & $A B$ & MUC1 & MUC2 & $M U C 5 A C$ & CA19-9 & CEA \\
\hline 1 & 12.7 & Multilocular & Adenoma & Gastric & $3+$ & $1+$ & $3+$ & - & - & $3+$ & - & - \\
\hline 2 & 20.0 & Multilocular & CIS in adenoma & Gastric & $3+$ & $2+$ & $3+$ & - & - & $3+$ & $2+$ & - \\
\hline 3 & 7.5 & Multilocular & CIS & Pancreaticobiliary & $3+$ & - & $2+$ & - & $1+$ & $3+$ & - & - \\
\hline 4 & 3.7 & Multilocular & CIS & Pancreaticobiliary & $3+$ & $1+$ & $3+$ & $2+$ & - & $3+$ & $3+$ & $2+$ \\
\hline 5 & 3.0 & Multilocular & CIS & Oncocytic & $3+$ & $2+$ & $3+$ & $1+$ & $2+$ & $3+$ & $1+$ & $1+$ \\
\hline 6 & 2.5 & Multilocular & CIS & Pancreaticobiliary & $3+$ & $2+$ & $3+$ & $1+$ & $2+$ & $3+$ & $2+$ & - \\
\hline 7 & 3.0 & Multilocular & CIS & Pancreaticobiliary & $3+$ & - & $3+$ & $2+$ & - & $3+$ & $1+$ & - \\
\hline 8 & 4.3 & Unilocular & Microinv mucinous ca & Pancreaticobiliary & $3+$ & $2+$ & $3+$ & $2+$ & $2+$ & $3+$ & - & - \\
\hline 9 & 8.2 & Multilocular & Microinv mucinous ca & Pancreaticobiliary & $3+$ & $1+$ & $2+$ & $1+$ & - & $3+$ & $1+$ & - \\
\hline Total & & & & & $100 \%$ & $78 \%$ & $100 \%$ & $67 \%$ & $44 \%$ & $100 \%$ & $67 \%$ & $22 \%$ \\
\hline
\end{tabular}

CIS, carcinoma in situ; microinv mucinous ca, microinvasive mucinous carcinoma; PAS, periodic acid-Schiff after diastase digestion; HID, highiron diamine; $\mathrm{AB}$, alcian blue, -, negative; $1+$, positive in $1-10 \%$ of tumor cells; $2+$, positive in $11-50 \%$ of tumor cells; $3+$, positive in more than $50 \%$ of tumor cells.

adenoma components. Cases 3, 4, 5, 6 and 7 were papillary adenocarcinoma in situ, whereas cases 8 and 9 were associated with focal microinvasion of mucinous carcinoma, which consisted histologically of extravasated mucin with floating carcinoma cells (Figure 6). The cyst wall was composed of fibrous connective tissue with focal lymphocytic infiltration or edematous change. Case 3 was associated with focal stromal hyalinization and calcification. Densely cellular connective tissue resembling ovarian stroma (ovarian-like stroma) was not observed in any cases.

Intraepithelial neoplasm was observed within non-dilated adjacent bile ducts in all cases, which suggested a luminal communication between cystic tumors and bile ducts and a continuous spread of neoplastic cells from cystic spaces to the surrounding bile ducts through this communication (Figure 7a). In case 4 , a direct communication between cystic tumor and non-neoplastic bile ducts was identified on histological specimens (Figure 7b). In addition, four cases (cases 3, 4, 6 and 9) had glandular tissues resembling peribiliary glands in the walls of the cystic tumors. Hepatic parenchyma was entrapped in the fibrous interlocular septa in three cases (cases 2, 4 and 6) (Figure 8). Based on these histological features, all cystic tumors used in this study were considered to have a luminal communication to the bile duct.

\section{Mucin Histochemistry}

The result of mucin histochemistry is shown in Table 2. Non-neoplastic biliary epithelium was diffusely positive for periodic acid-Schiff after diastase digestion and high-iron diamine, and focally positive for alcian blue, the pattern of which corresponded predominantly to neutral mucin and 

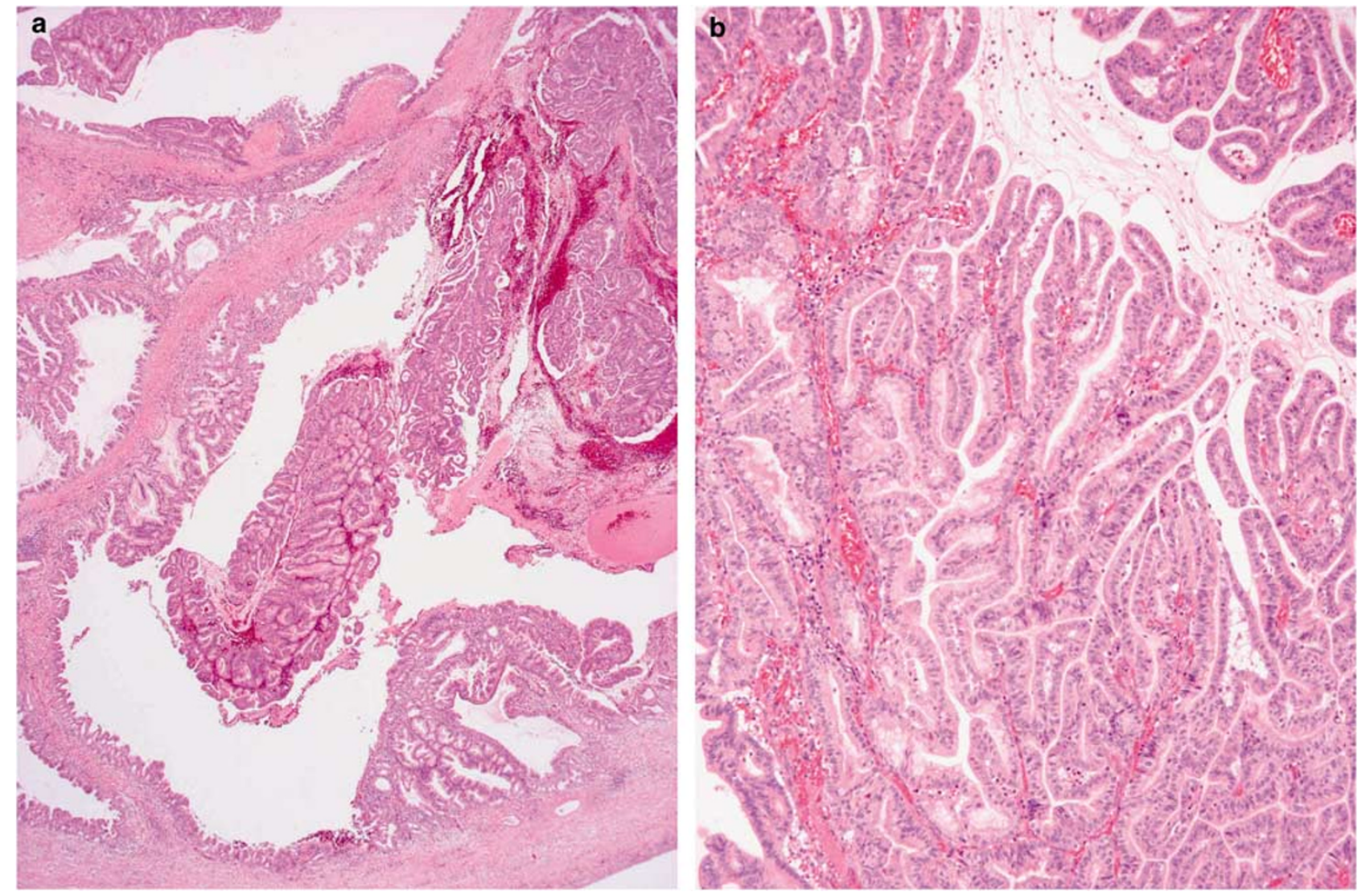

Figure 3 Microscopic features of biliary cystic tumor. Intracystic papillary proliferation with fibrovascular cores is observed (case 6, HE staining, a: $\times 20, \mathbf{b}: \times 40$ ).
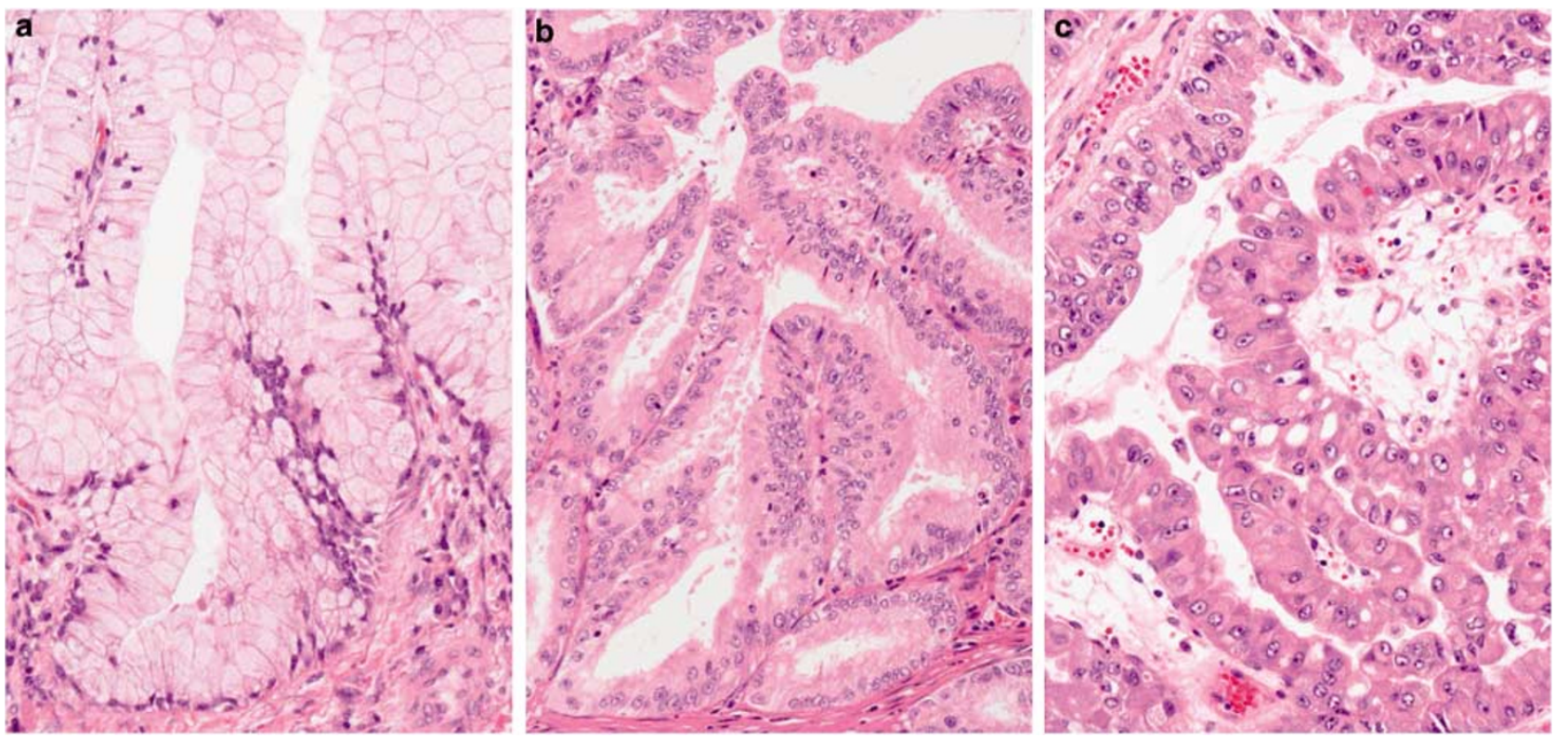

Figure 4 Three tumor cell types observed in biliary cystic tumors. Tumor cells correspond to the gastric type (a), the pancreaticobiliary type (b), and the oncocytic type (c) of IPMN-P (a: case 1, b: case 4, c: case 5, HE staining, $\times 400$ ).

sulfomucin. Biliary cystic tumors were commonly positive for alcian blue and periodic acid-Schiff after diastase digestion, with the reduced expression of high-iron diamine. That is, sialomucin expression was increased in biliary cystic tumors compared to non-neoplastic biliary epithelium. 


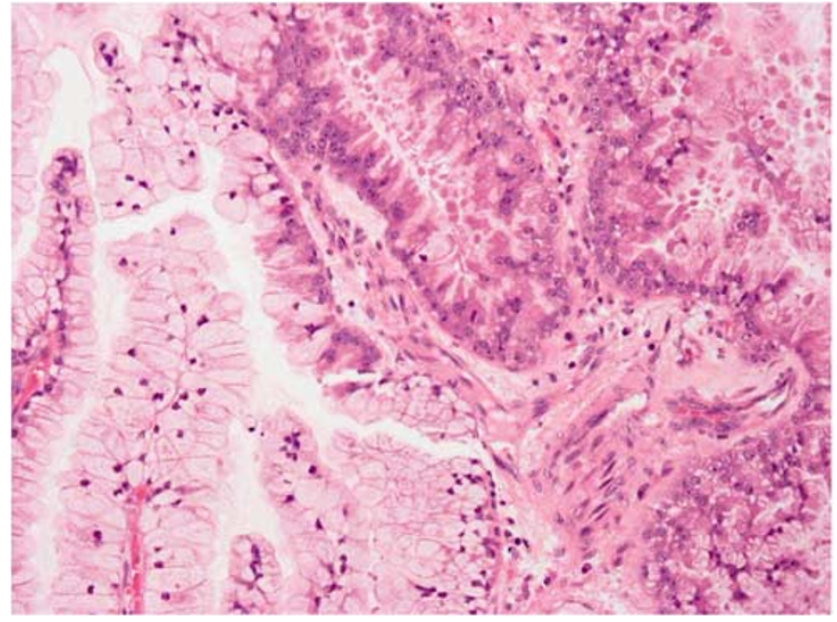

Figure 5 Histopathology of biliary cystic tumor (adenocarcinoma in adenoma). This tumor consists of adenoma (left) and noninvasive adenocarcinoma components (right). Front formation is observed between these two components (case 2, H\&E staining, $\times 200$ ).

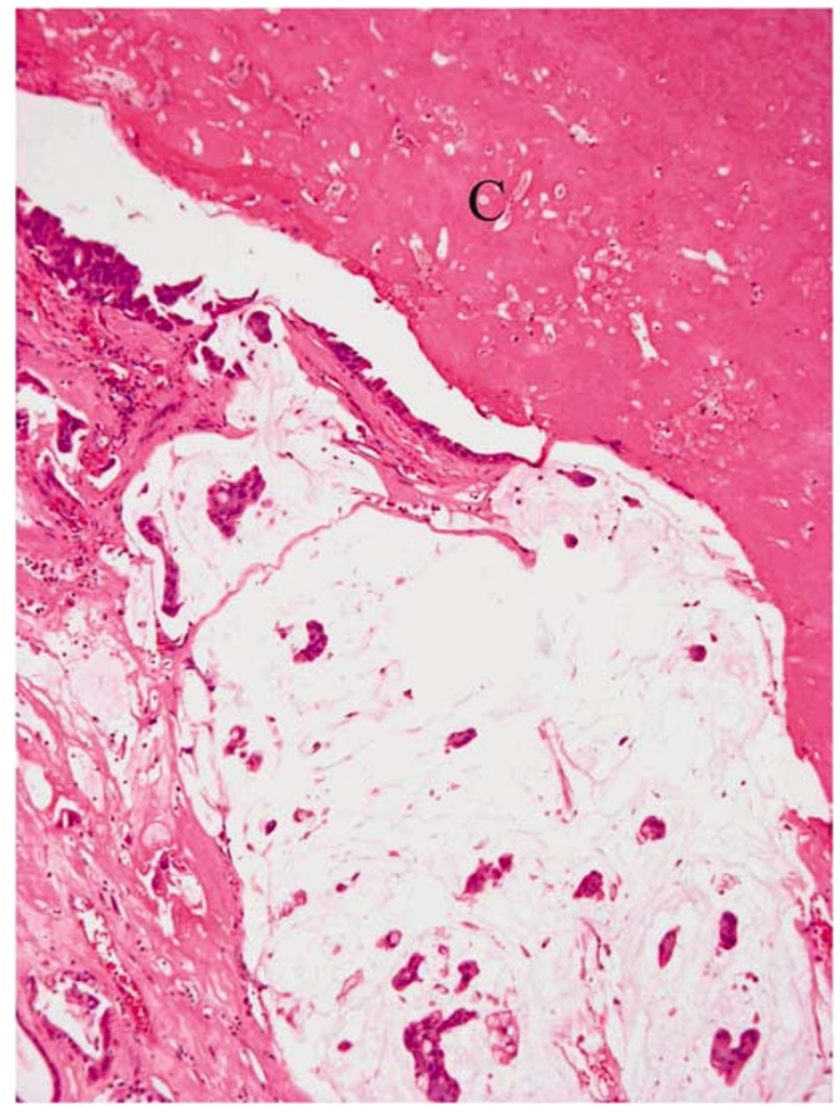

Figure 6 Microinvasive mucinous adenocarcinoma observed in biliary cystic tumor. Fibrinous exudate is observed in the intracystic space (C) (case 9, H\&E staining, × 100).

\section{Immunohistochemistry}

The result of immunohistochemistry is shown in Table 2. MUC1 was expressed in the apical membrane of tumor cells. MUC2 and MUC5AC were mostly expressed in the cytoplasm of tumor cells. Two cases of the gastric type (cases 1 and 2) were both negative for MUC1 and MUC2. The pancreaticobiliary (cases 3, 4, 6, 7, 8 and 9) and oncocytic types (case 5) commonly expressed MUC1 and MUC2. The diffuse expression of MUC5AC was found in all cases, irrespective of the tumor cell types. Invasive lesions of cases 8 and 9 showed expression patterns of MUC1, MUC2 and MUC5AC, similar to those of non-invasive lesions. Double immunostaining of MUC2 and CDX2 revealed double-positive tumor cells in all four cases with MUC2 expression on single immunostaining (Figure 9).

CA19-9 expression was observed in six cases (cases 2, 4, 5, 6, 7 and 9). CA19-9 was expressed not only in adenocarcinoma, but also the adenoma component in case 2. In case 9, CA19-9 expression was focally observed only in the non-invasive lesion, not in the invasive lesion, probably due to the small size of the invasive lesion. CEA expression was observed in two cases (cases 4 and 5), although its expression was only focal in case 5 . No stromal cells with the nuclear expression of ER or PgR were observed in any cases.

\section{Prognosis after Surgical Resection}

The survival status at the last medical follow-up is shown in Table 1 . One patient (case 7) died in direct operative mortality 30 days after surgery. The remaining eight cases were alive without any tumor recurrences (follow-up period: 3-156 months).

\section{Discussion}

In this study, we examined the clinicopathological features of biliary cystic tumors with bile duct communication. Radiologically, we examined the luminal communication with ERC or intraoperative direct cholangiography, because CT or MRI imaging is insufficient to examine the luminal communication of biliary cystic tumors with bile ducts. It was found in this study that five cases of biliary cystic tumors showed a luminal communication on cholangiographies. Especially, intraoperative cholangiography was useful to examine a ductal communication, because both of two cases examined were found to have a ductal communication. Macroscopically, such communication could not be demonstrated clearly in any cases, probably because the ductal communication was too narrow or twisted to identify on gross examination. Histologically, intraductal spreading of tumor cells along the pre-existing bile duct outside cystic tumors was observed in all cases, and this finding was consistent with there being a luminal communication between cystic tumors and the bile duct. We thought that tumor spreading into the bile duct could not occur without any direct communication, because 

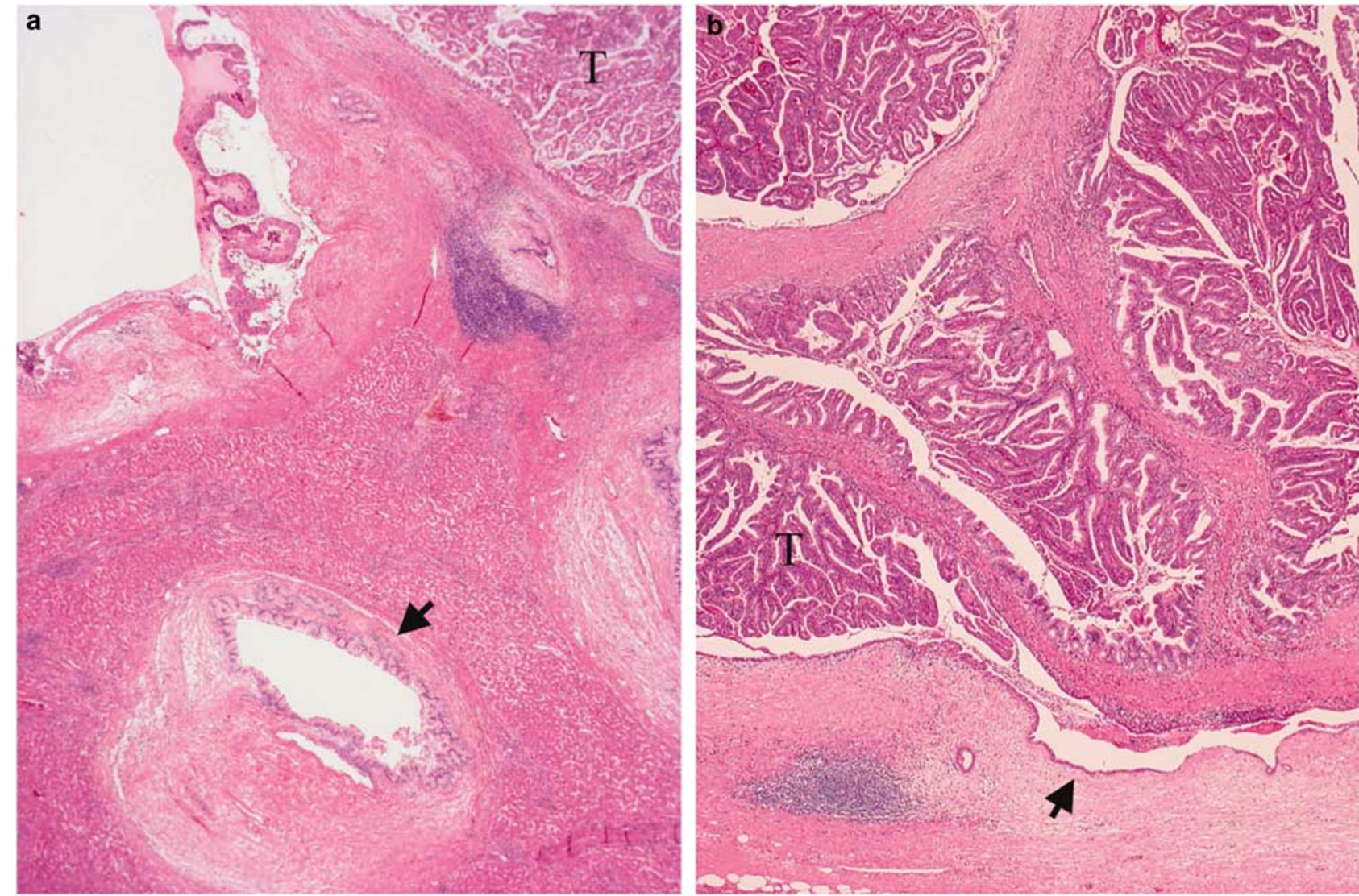

Figure 7 Intraductal spreading of neoplastic epithelium into adjacent bile ducts. (a) Intraductal papillary tumor is observed in the intrahepatic bile duct (arrows) around biliary cystic tumor (T). (b) A direct luminal communication between cystic tumor (T) and nonneoplastic bile duct (arrow) is identified (case 4, H\&E staining, $\times 20$ ).

all cases examined in this study were non-invasive or microinvasive tumors. In addition, it was interesting that some cases were associated with glandular tissues resembling peribiliary glands in the cyst walls (four cases) or hepatic parenchyma in the interlocular septa (three cases). These histological features suggested that some of the cystic spaces in those cases were derived from the cystic dilatation of pre-existing bile ducts. Associations with glandular tissues or entrapped hepatic parenchyma have not been reported in biliary cystic tumor to the best of our knowledge, and they might be useful findings favoring a luminal communication of biliary cystic tumors with the bile ducts.

In 2001, we reported that a characteristic intraductal papillary neoplasm (IPN-B) could occur in the intrahepatic bile duct associated with hepatolithiasis in East Asia. ${ }^{16}$ After that, great attention was drawn to IPN-B as a biliary counterpart of IPMN-P. ${ }^{18-20,25}$ The clinicopathological characteristics of IPN-B can be summarized as follows: ${ }^{16,19,20,26}$ (1) prominent intraductal papillary proliferation with local dilatation of the bile duct, (2) the acquisition of gastro-intestinal phenotypes, such as MUC2, MUC5AC and CDX2 expressions, (3) mucin hypersecretion, (4) a common association with invasive mucinous carcinoma and (5) favorable prognosis compared to conventional cholangiocarcinoma. Biliary cystic tumors examined in this study shared all of these pathological characteristics. It might be easily understandable that IPN-B could manifest cystic tumors after cystic ductal dilatation with prominent intraductal mucin accumulation.

IPMN-P can be histologically classified into four based on tumor cell types: pancreaticobiliary, intestinal, gastric and oncocytic types. ${ }^{21-24}$ Clinicopathological differences among them have been reported. ${ }^{21-23}$ Adenoma is more common in the gastric type, whereas the pancreaticobiliary, intestinal and oncocytic types are mostly associated with adenocarcinoma. ${ }^{27}$ Similarly, in the present study, two cases of the gastric type had adenoma components, whereas all cases of the pancreaticobiliary and oncocytic types were adenocarcinoma with or without invasive lesions. In IPMN-P, the gastric type is immunohistochemically characterized by negativity for MUC1 and MUC2, whereas the pancreaticobiliary and oncocytic types commonly expresses MUC1 in addition to the occasional expression of MUC2. ${ }^{21-23}$ These immunophenotypes resembled those of gastric, pancreaticobiliary and oncocytic 


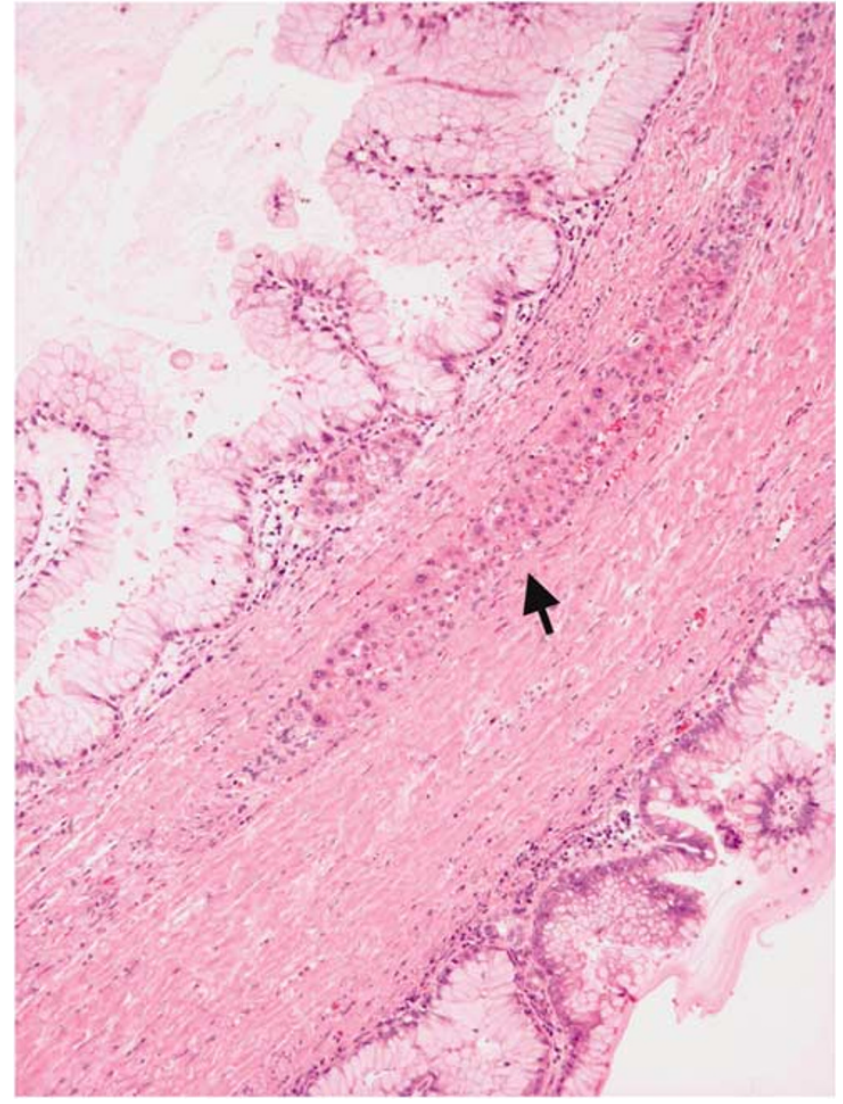

Figure 8 Hepatic parenchyma is observed in interlocular fibrous septa of biliary cystic tumor (arrow) (case 2, H\&E staining, $\times 100$ ).

types of biliary cystic tumors examined in this study. Common phenotypic changes might occur during tumorigenesis or tumor progression in both IPMN-P and biliary cystic tumor with ductal communication. In addition, it is interesting that the intestinal type tumor could not be observed in this study, because the intestinal type is the most common in IPN-B associated with hepatolithiasis. ${ }^{20}$ It is plausible that the tumor cell type of IPN-B might affect the growth types of tumor (cystic or local dilatation), or reflect the preceding condition (association with hepatolithiasis or not). However, additional research on multiple IPN-B cases of the cystic type is indispensable in order to clarify this issue.

Interestingly, the biliary cystic tumors examined in this study did not have ovarian-like stroma. Wheeler and Edmondson reported the clinicopathological characteristics of 17 cases of biliary cystic tumor with ovarian-like stroma in $1985 .{ }^{3}$ We compared and summarized the clinicopathological differences between the present cases and Wheeler's cases in Table 3. Biliary cystic tumors with ovarianlike stroma occurred only in females, whereas our cases consisted of both genders. Patients with biliary cystic tumor with ovarian-like stroma were younger than our cases. The prevalence of tumor locations (intrahepatic) and the type of cyst (multilocular

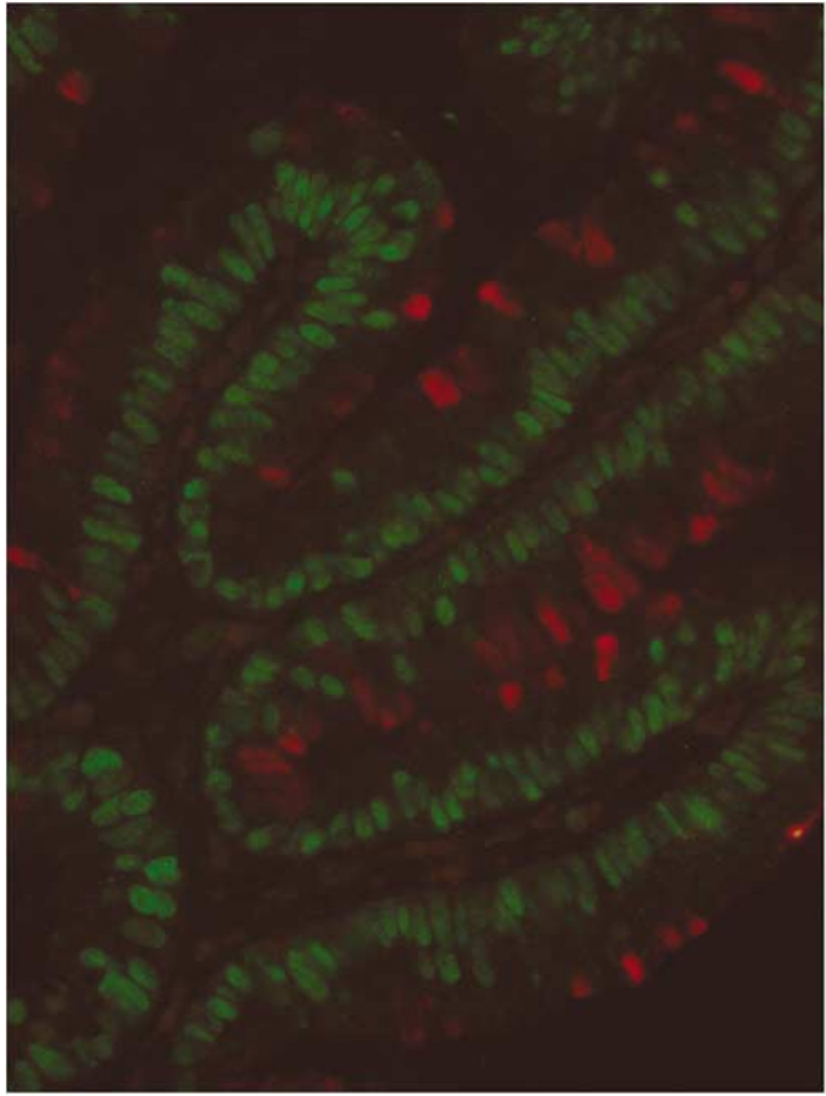

Figure 9 Double immunostaining of MUC2 and CDX2 reveals the cytoplasmic expression of MUC2 (red) and the nuclear expression of CDX2 (green) in some tumor cells (case $6, \times 400$ ).

cyst) were similar in both tumor groups. Biliary cystic tumor with ovarian-like stroma was larger than our cases, although this difference might be caused by the fact that Wheeler's cases were earlier than ours. Recent advances in imaging modalities might contribute to the early detection of biliary tumors. The luminal communication with the bile duct was not sufficiently examined in Wheeler's case, although almost all cases of biliary cystic tumor with ovarian-like stroma could not be found to have ductal communication until now with minor exceptions. ${ }^{28}$ That is, our cases had a ductal communication and did not have ovarian-like stroma, and could occur in both genders. In contrast, biliary cystic tumors with ovarian-like stroma usually do not have a ductal communication, and can only occur in female patients. Based on these pathological characteristics, biliary cystic tumors with ductal communication or ovarian-like stroma might correspond to IPMN-P or mucinous cystic neoplasms of the pancreas, respectively. We speculate that the term of biliary cystadenoma or cystadenocarcinoma should be restricted to true cystic neoplasms with ovarian-like stroma, same as in the pancreas.

In addition, it is interesting that benign lesions (adenoma) were more common in biliary cystic 
Table 3 Comparison of clinicopathological features between the present cases and biliary cystic tumor with mesenchymal stroma (ovarian-like stroma)

\begin{tabular}{|c|c|c|c|}
\hline & $\begin{array}{c}\text { Cystic tumors with bile duct } \\
\text { communication } \\
\text { (9 cases })\end{array}$ & $\begin{array}{c}\text { Cystic tumors with } \\
\text { mesenchymal stroma } \\
\text { (17 cases) }\end{array}$ & \\
\hline Gender & & & $P=0.0008$ \\
\hline Male & $5(56 \%)$ & 0 & \\
\hline Female & $4(44 \%)$ & $17(100 \%)$ & \\
\hline Average age (years) & $67.0(52-84)$ & $45.6(19-67)$ & $P=0.0007$ \\
\hline Location & & & $P=0.9624$ \\
\hline Intrahepatic & $8(89 \%)$ & $16(88 \%)$ & \\
\hline Hepatic hilum & $1(11 \%)^{\mathrm{b}}$ & $1(6 \%)^{c}$ & \\
\hline Extrahepatic & 0 & $1(6 \%)^{\mathrm{c}}$ & \\
\hline Type of cyst & & & $P=0.3015$ \\
\hline Multilocular & $8(89 \%)$ & $12(71 \%)$ & \\
\hline Unilocular & $1(11 \%)$ & $5(29 \%)$ & \\
\hline Tumor size $(\mathrm{cm})$ & $6.1(2.5-20.0)$ & $16.7(7.0-25.0)^{\mathrm{d}}$ & $P=0.0001$ \\
\hline Histopathology & & & $P=0.0018$ \\
\hline Adenoma & $1(11 \%)$ & $13(76 \%)$ & \\
\hline Adenocarcinoma & $8(89 \%)$ & $4(24 \%)$ & \\
\hline Epithelial type & & & Identical \\
\hline Mucinous & $9(100 \%)$ & $17(100 \%)$ & \\
\hline Serous & 0 & 0 & \\
\hline Ovarian-like stroma & None & Present & \\
\hline $\begin{array}{l}\text { Luminal communication with } \\
\text { surrounding bile ducts }\end{array}$ & Present & Not identified & \\
\hline
\end{tabular}

${ }^{\mathrm{a}}$ Referring to Wheeler et $a l^{3}$.

${ }^{\mathrm{b}}$ Case 9 having a communication to right hepatic duct.

${ }^{\mathrm{C}}$ Two lesions in the same case.

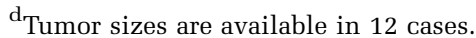

tumors with ovarian-like stroma. Out of nine cases examined in this study, six were adenocarcinoma in situ and two cases were microinvasive mucinous carcinoma (Tables 2 and 3). According to Devaney's report, which was the largest study of biliary cystic tumors, 52 out of 70 cases $(74 \%)$ were histologically adenoma and 18 cases (26\%) were adenocarcinoma. ${ }^{4}$ Ovarian-like stroma was observed in $85 \%$ (44 of 52 cases) of biliary adenoma cases and 28\% (five of 18 cases) of adenocarcinoma cases. Ishak et al also reported that ovarian-like stroma was observed in seven of eight cases of benign biliary cystic tumor (adenoma), but not identified in any adenocarcinoma cases (six cases). ${ }^{2}$ That is, most cases of biliary cystic tumor with ovarian-like stroma were adenoma, whereas those without ovarian-like stroma were more commonly adenocarcinoma. It seems plausible that some biliary cystic tumors classified as biliary cystadenoma and cystadenocarcinoma without ovarian-like stroma in the previous reports might have a luminal communication with the bile duct, and could be a cystic variant of IPN-B, same as the present cases.

Recent recognition of the disease entity of IPN-B would create some confusion on their discrimination from other biliary tumors. From the view point of papillary proliferation, discrimination between
IPN-B and biliary papillomatosis or cholangiocarcinoma of intraductal growth-type might be a problem. ${ }^{29-33}$ We now speculate that most of biliary papillomatosis and cholangiocarcinoma of intraductal growth-type can be regarded as IPN-B, although a detailed clinicopathological study using a large numbers of cases is mandatory to conclude this issue. Biliary tumors with abundant mucin production have been called mucin-hypersecreting bile duct tumors. Kim et $a l^{34}$ and Shibahara et $a l^{17}$ reported a homology between mucin-hypersecreting bile duct tumor and IPMN-P from the radiological and pathological standpoints, respectively. We think that IPN-B has been called by different names until now based on predominant pathological features such as biliary cystadenoma/adenocarcinoma (cystic proliferation), biliary papillomatosis (multiplicity) and mucin-hypersecreting bile duct tumor (mucin over-production). Kinds of genetic or phenotypic alteration might influence on different manifestations of IPN-B attracts our interest.

In conclusion, we examined the clinicopathological features of biliary cystic tumors with communication to the bile duct. The obtained results suggested biliary cystic tumor with bile duct communication might be a cystic variant of IPN-B rather than true biliary cystic neoplasms. 


\section{References}

1 Wittekind C, Fischer HP, Ponchon T. Bile duct cystadenoma and cystadenocarcinoma. In: Hamilton SR, Aaltonen LA (eds). World Health Organization Classification of Tumours. Pathology and Genetics of Tumours of the Digestive System. IARC Press: Lyon, France, 2000, pp 182-183.

2 Ishak KG, Willis GW, Cummins SD, et al. Biliary cystadenoma and cystadenocarcinoma: report of 14 cases and review of the literature. Cancer 1977;39:322-338.

3 Wheeler DA, Edmondson HA. Cystadenoma with mesenchymal stroma (CMS) in the liver and bile ducts. A clinicopathologic study of 17 cases, 4 with malignant change. Cancer 1985;56:1434-1445.

4 Devaney K, Goodman ZD, Ishak KG. Hepatobiliary cystadenoma and cystadenocarcinoma. A light microscopic and immunohistochemical study of 70 patients. Am J Surg Pathol 1994;18:1078-1091.

5 Sato M, Watanabe Y, Tokui K, et al. Hepatobiliary cystadenocarcinoma connected to the hepatic duct: a case report and review of the literature. Hepatogastroenterology 2003;50:1621-1624.

6 Hanazaki K. Intrahepatic biliary cystadenoma demonstrated by intraoperative cholangiography. Hepatogastroenterology 1996;43:1024-1028.

7 Frameglia M, Nimura Y, Hayakawa N, et al. Biliary cystadenocarcinoma resected by segment 3 and 4 hepatectomy. Hepatogastroenterology 1996;43:1029-1034.

8 Zamboni G, Kloppel G, Hruban RH, et al. Mucinous cystic neoplasms of the pancreas. In: Hamilton SR, Aaltonen LA (eds). World Health Organization Classification of Tumours. Pathology and Genetics of Tumours of the Digestive System. IARC Press: Lyon, France, 2000, pp 234-236.

9 Longnecker DS, Adler G, Hruban RH, et al. Intraductal papillary-mucinous neoplasms of the pancreas. In: Hamilton SR, Aaltonen LA (eds). World Health Organization Classification of Tumours. Pathology and Genetics of Tumours of the Digestive System. IARC Press: Lyon, France, 2000, pp 237-240.

10 Longnecker DS. Observations on the etiology and pathogenesis of intraductal papillary-mucinous neoplasms of the pancreas. Hepatogastroenterology 1998; 45:1973-1980.

11 Ohhashi K, Murakami Y, Maruyama M. Four cases of mucous secreting pancreatic cancer. Prog Dig Endosc 1982;20:348-351.

12 Zamboni G, Scarpa A, Bogina G, et al. Mucinous cystic tumors of the pancreas: clinicopathological features, prognosis, and relationship to other mucinous cystic tumors. Am J Surg Pathol 1999;23:410-422.

13 Suzuki Y, Atomi Y, Sugiyama M, et al. Japanese multiinstitutional study of intraductal papillary mucinous tumor and mucinous cystic tumor. Cystic neoplasm of the pancreas: a Japanese multiinstitutional study of intraductal papillary mucinous tumor and mucinous cystic tumor. Pancreas 2004;28: 241-246.

14 Compagno J, Oertel JE. Mucinous cystic neoplasms of the pancreas with overt and latent malignancy (cystadenocarcinoma and cystadenoma). A clinicopathologic study of 41 cases. Am J Clin Pathol 1978;69: $573-580$.

15 Thompson LD, Becker RC, Przygodzki RM, et al. Mucinous cystic neoplasm (mucinous cystadenocarcinoma of low-grade malignant potential) of the pancreas: a clinicopathologic study of 130 cases. Am J Surg Pathol 1999;23:1-16.

16 Chen TC, Nakanuma Y, Zen Y, et al. Intraductal papillary neoplasia of the liver associated with hepatolithiasis. Hepatology 2001;34:651-658.

17 Shibahara H, Tamada S, Goto $\mathrm{M}$, et al. Pathologic features of mucin-producing bile duct tumors: two histopathologic categories as counterparts of pancreatic intraductal papillary-mucinous neoplasms. Am J Surg Pathol 2004;28:327-338.

18 Abraham SC, Lee JH, Boitnott JK, et al. Microsatellite instability in intraductal papillary neoplasms of the biliary tract. Mod Pathol 2002;15:1309-1317.

19 Shimonishi $\mathrm{T}$, Zen Y, Chen TC, et al. Increasing expression of gastrointestinal phenotypes and p53 along with histologic progression of intraductal papillary neoplasia of the liver. Hum Pathol 2002;33: 503-511.

20 Zen Y, Sasaki M, Fujii T, et al. Different expression patterns of mucin core proteins and cytokeratins during intrahepatic cholangiocarcinogenesis from biliary intraepithelial neoplasia and intraductal papillary neoplasm of the bile duct-an immunohistochemical study of 110 cases of hepatolithiasis. J Hepatol 2006; 44:350-358.

21 Adsay NV, Conlon KC, Zee SY, et al. Intraductal papillary-mucinous neoplasms of the pancreas: an analysis of in situ and invasive carcinomas in 28 patients. Cancer 2002;94:62-77.

22 Luttges J, Zamboni G, Longnecker D, et al. The immunohistochemical mucin expression pattern distinguishes different types of intraductal papillary mucinous neoplasms of the pancreas and determines their relationship to mucinous noncystic carcinoma and ductal adenocarcinoma. Am J Surg Pathol 2001;25: 942-948.

23 Yonezawa S, Nakamura A, Horinouchi $\mathrm{M}$, et al. The expression of several types of mucin is related to the biological behavior of pancreatic neoplasms. J Hepatobiliary Pancreat Surg 2002;9:328-341.

24 Furukawa T, Kloppel G, Volkan Adsay N, et al. Classification of types of intraductal papillary-mucinous neoplasm of the pancreas: a consensus study. Virchows Arch 2005;447:794-799.

25 Abraham SC, Lee JH, Hruban RH, et al. Molecular and immunohistochemical analysis of intraductal papillary neoplasms of the biliary tract. Hum Pathol 2003; 34:902-910.

26 Ishikawa A, Sasaki M, Ohira S, et al. Aberrant expression of CDX2 is closely related to the intestinal metaplasia and MUC2 expression in intraductal papillary neoplasm of the liver in hepatolithiasis. Lab Invest 2004;84:629-638.

27 Nakamura A, Horinouchi M, Goto $\mathrm{M}$, et al. New classification of pancreatic intraductal papillary-mucinous tumour by mucin expression: its relationship with potential for malignancy. J Pathol 2002;197:201-210.

28 Albores-Saavedra J, Henson DE, Klimstra DS (eds). Tumors of the gallbladder, extrahepatic bile ducts, and ampulla of Vater, 3rd edn. AFIP: Washington, DC, 2000, 174pp.

29 Lee SS, Kim MH, Lee SK, et al. Clinicopathologic review of 58 patients with biliary papillomatosis. Cancer 2004;100:783-793.

30 Martin RC, Klimstra DS, Schwartz L, et al. Hepatic intraductal oncocytic papillary carcinoma. Cancer 2002;95:2180-2187. 
31 Yeh TS, Tseng JH, Chen TC, et al. Characterization of intrahepatic cholangiocarcinoma of the intraductal growth-type and its precursor lesions. Hepatology 2005;42:657-664.

32 Sudo Y, Harada K, Tsuneyama K, et al. Oncocytic biliary cystadenocarcinoma is a form of intraductal oncocytic papillary neoplasm of the liver. Mod Pathol 2001;14:1304-1309.
33 Kloppel G, Kosmahl M. Is the intraductal papillary mucinous neoplasia of the biliary tract a counterpart of pancreatic papillary mucinous neoplasm? J Hepatol 2006;44:249-250.

34 Kim HJ, Kim MH, Lee SK, et al. Mucin-hypersecreting bile duct tumor characterized by a striking homology with an intraductal papillary mucinous tumor (IPMT) of the pancreas. Endoscopy 2000;32:389-393. 